\title{
Performance analysis of graphene modified porous oil retainer materials
}

\author{
NIU Rong Jun"1,2,a, Zhang Jian Hü,b, Ni Yan Guang ${ }^{2, c}$ and Deng Si Er ${ }^{1}$ \\ ${ }^{1}$ School of Mechatronics Engineering, Henan University of Science and Technology, Luoyang \\ 471003, China \\ ${ }^{2}$ National United Engineering Laboratory for Advanced Bearing Tribology, Henan University of \\ Science and Technology, Luoyang 471132, China \\ aniurj@haust.edu.cn, b1536260009@qq.com, nyg321@126.com
}

\begin{abstract}
Keywords: rolling bearing, retainer, porous oil material, friction and wear performance Abstract: Porous oil retainer is a key factor to ensure good lubrication of the inertial navigation bearings. The PTFE/PI porous retainer made of the original recipe and process preparation, in the high-speed operation, will appear serious wear, high temperature rise, a large amount of oil loss in the micro pores due to expansion and other issues. These lead to the lubrication life greatly reduced. It cannot meet the high-speed and long-time operation requirements of high-precision bearings. In this paper, a improved porous retainer composite is developed, which is using porous PI as the matrix , graphene as the enhancing modified additives, and PTFE as lubricating modified additives. The improved porous retainer composite is prepared by using the constant volume sintering method. The effect of graphene addition on the mechanical properties and tribological properties of porous retainer composites is studied. The results show that the high strength properties of graphene and the good wear resistance performance of PTFE improve the porous polyimide retainer composites.
\end{abstract}

\section{Introduction}

The porous retainer material is based on synthetic polymer materials, and uses special ways to form interconnected pores inside the material, so that it not only has enough mechanical properties, but also has strong ability to store and release lubricating oil ${ }^{[1-4]}$.

Currently, the porous polyimide retainer is a polyimide matrix, adding appropriate amount of PTFE, using a special molding process. Because PTFE has excellent heat resistance and good self-lubricating, the friction coefficient of PTFE modified polyimide composites compared to pure polyimide is reduced by about $34 \%{ }^{[3-4]}$. However, due to poor wear resistance of PTFE, the wear resistance of the modified porous polyimide composite material becomes worse. During the high-speed operation, the porous retainer of the bearing will be serious wear and tear, higher temperature and large loss of lubricating oil due to expansion, which will greatly reduce the lubrication life, and can not meet the requirements of some high speed bearings ${ }^{[5-6]}$. Therefore, to meet the need of high precision bearings for long and high speed work, it is very important to improve the wear resistance of the porous retainer material.

In recent years, a large number of researchers have studied the tribological properties of the microscopic properties of graphene and the tribological properties of the macroscopic properties ${ }^{[7-12]}$. If the rigidity and thermal conductivity of the graphene are combined with the toughness and the workability of polyimide, graphene as a filler will significantly enhance and improve the mechanical and tribological properties of composites.

In this paper, porous PI as the matrix, graphene as the enhancing modified additives, PTFE as lubricating modified additives, the improved porous retainer composite is prepared by using the constant volume sintering method. The effects of graphene addition on mechanical properties and tribological properties of porous retainer composites are studied, so as to meet the increasingly demanding working conditions. 


\section{Preparation and forming process of reinforced porous retainer ring billet material}

The reinforced porous retainer material is based on polyimide material, and its preparation process is taking constant volume sintering method. According to the required material density of the retainer by calculation, the raw materials are loaded into the mold in the process of sintering, then put some pressure on the raw materials to overcome the inherent viscoelastic properties of polyimide materials.

The polyimide, graphene and PTFE powder are dried in the blast dryer for more than four hours at $120^{\circ} \mathrm{C}$, and the polyimide powder is sieved to make the polyimide powder reach 80 200 mesh size. The electronic balance is used to weigh modified additives and polyimide powder with different mass ratio. Stir with high speed agitator and repeat for more than three times to ensure the polyimide composite powder mixed evenly. The polyimide composite powder with a certain mass is loaded into the mold, and compacted by $2 \mathrm{kN} / \mathrm{cm}^{2} \sim 8 \mathrm{kN} / \mathrm{cm}^{2}$ on the hydraulic press. The mould is locked tightly with the locking force of $100 \mathrm{~N} \sim 500 \mathrm{~N}$ by the fixture. The molds (including fixtures) placed in a rotary blast furnaceon sinter at 370 to 390 degree temperature, and then insulate for 5 to 40 minutes, cool and demould finally. The ring-shaped samples of PTFE modified PI and graphene modified PTFE/PI are prepared as shown in Fig.1.

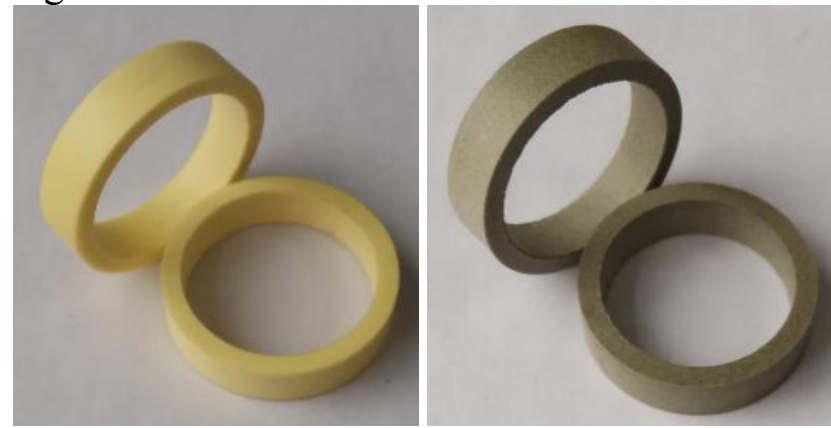

Figure 1 Ring-type samples of different recipe preparation composites

\section{Enhancement performance analysis of porous retainer material}

\section{Thermal conductivity}

PTFE modified polyimide and graphene modified PTFE/PI are made into three samples, respectively, and the thermal conductivity of the samples is tested by using laser thermal conductivity meter(LFA) under the condition of 60 degree temperature and relative humidity less than $50 \%$. The test results are shown in table 1.

\begin{tabular}{ccc} 
Table 1 Thermal conductivity of porous composite materials $\left(\mathrm{T}=60^{\circ} \mathrm{C}\right.$ & \\
\hline composites & thermal conductivity, $\mathrm{W} /(\mathrm{m} \cdot \mathrm{K})$ & \\
& 0.29 & 0.287 \\
PTFE+PI & 0.28 & \\
& 0.29 & 0.393 \\
GP+PTFE+PI & 0.40 & \\
& 0.39 &
\end{tabular}

It can be seen from table 1 that the thermal conductivity of PTFE/PI composites modified by graphene is $37 \%$ higher than that of PTFE modified PI composites. This is due to the excellent thermal conductivity of the graphene itself. When it is embedded in the composite, the thermal conductivity of the composites is improved. However, the thermal conductivity of composites is not improved obviously. The main reason is that graphene is well embedded in the polyimide composite material, but it doesn't form continuous phase completely, thus affecting the improvement of its thermal conductivity.

\section{Tensile strength of ring blank specimen}

In order to simulate the actual application state, the composites are made into a ring shape, and the tensile strength properties of the porous polyimide composites are tested by universal material testing machine at room temperature of $25 \pm 3$ degree, relative humidity of environment less than $50 \%$, and testing speed of $5 \sim 15 \mathrm{~mm} / \mathrm{min}$. The tensile specimen of the ring samples are shown in Fig.1. 
Fig.2 shows the tensile strength comparison of polyimide (GP+PI) and modified $\mathrm{PTFE} / \mathrm{PI}(\mathrm{GP}+\mathrm{PTFE}+\mathrm{PI})$ specimen with different graphene proportion components.

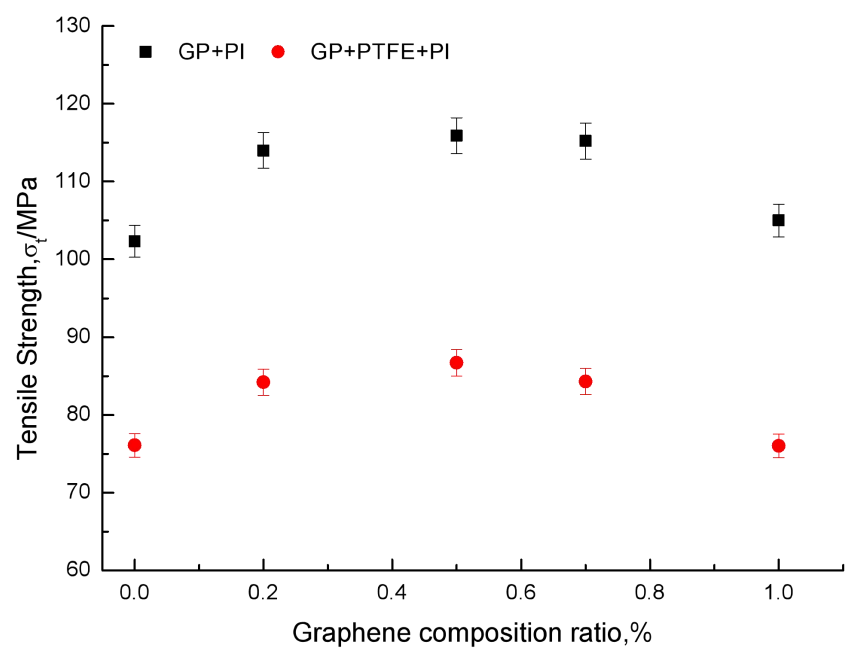

Figure 2 Tensile strength of ring blank specimens

From Fig.2, it can be seen that the tensile strength properties of the two modified porous polyimide composites firstly increase and then decrease with the increase of the proportion of graphene, and the tensile strength attain maximum when the ratio of graphene is $0.5 \%$. This is because it forms the optimum embedding ratio between graphene and polyimide powders when the proportion of graphene is $0.5 \%$, which improves the density of composites, and makes the tensile strength to reach the maximum. As the proportion of graphene increases, it affects the bond between the matrix and the tensile strength begins to decrease.

\section{The friction and wear properties of the retainer material}

The tribological properties of the sample materials are tested on a high speed reciprocating friction and wear tester(HSR-2M). Test conditions: reciprocating dry friction pair, steel ball diameter of $4 \mathrm{~mm}$, the test load 40 Newton, reciprocating line speed $100 \mathrm{~mm} / \mathrm{min}$, reciprocating distance $4 \mathrm{~mm}$ (bidirectional), test time per sample $10 \mathrm{~min}$.

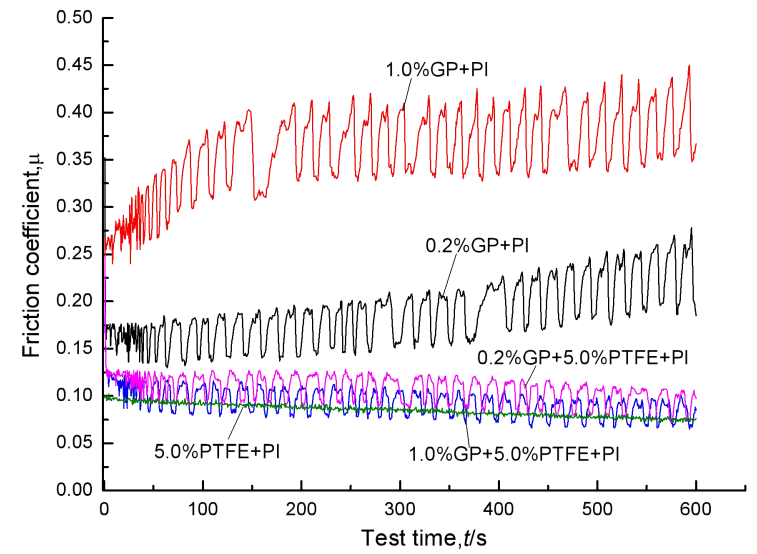

Figure 3 Dynamic variation curve of friction coefficient

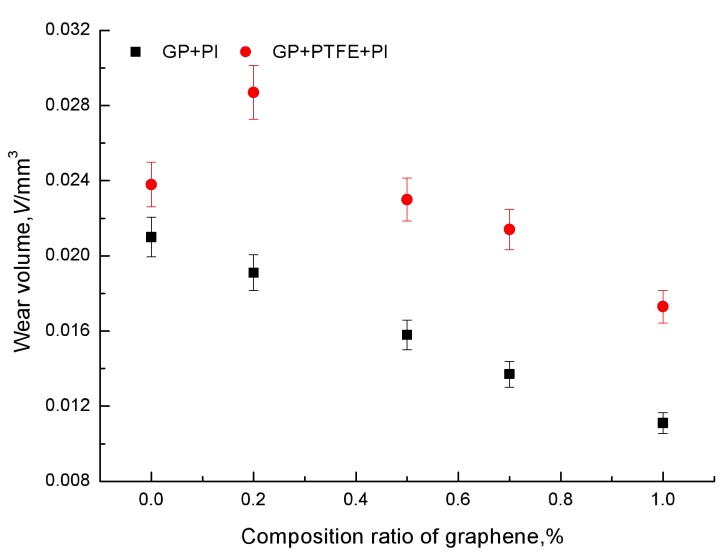

Figure 4 Effect of graphene ratio on wear volume of Porous composites

Fig. 3 shows the friction coefficient-time curves of porous materials with different proportions of Graphene, PTFE and PI. It can be seen that the friction coefficient fluctuates in a certain range due to the reciprocating movement of the grinding ball and the specimen. The friction coefficient of graphene modified polyimide material is obviously higher than that of graphene modified PTFE/PI material. The friction coefficient fluctuation range of the graphene modified polyimide increases with the increase of the proportion of graphene, and it needs to take a long time to reach the stable state. However, the friction coefficient of graphene modified PTFE/PI rapidly decreases, and it quickly reaches a stable state and remains in a certain range. 
In Fig.6, it can be seen that with the increase of the proportion of graphene, the wear volume of the modified polyimide composites decreases gradually. When the addition ratio of graphene is $1 \%$, the wear volume of the modified polyimide composite is reduced by about $50 \%$. This is due to the increase of the surface smoothness of polyimide composites after adding graphene, and the rigidity of graphene under micro-perspective, which makes the wear volume of composites decrease gradually. However, the wear volume of the graphene modified PTFE/PI composites shows a tendency to increase first and then decrease. This is due to the fact that when the proportion of graphene is relatively small, the matrix surface strength properties of the composites are poor, so the wear volume increases, and with the increase of the proportion of graphene, the strength properties of the matrix surface increase, so the wear volume begins to decrease.

The data analysis shows that the friction and wear properties of the graphene modified PTFE/PI composite have been improved, the wear volume decrease by about $27 \%$.

\section{Friction and Wear Mechanism of Porous retainer Materials}

The worn porous traces of the tested composite materials are placed under a high magnification microscope and photographed at $\times 200$ and $\times 500$ times, respectively. The comparison results are shown in Fig.5.
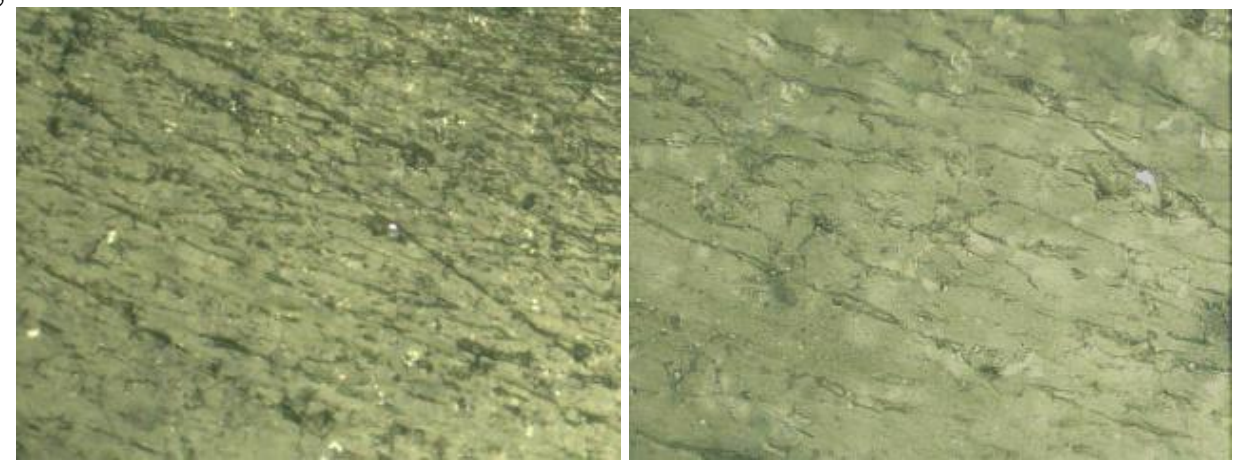

Figure 5 Micro wear morphology of Porous composites

Graphene modified PTFE/PI porous material has a higher level of smoothness and shallow grinding trail channel. The graphene has high strength properties and can bear most of the load in composite porous materials. The PTFE crystal has the characteristics of being embedded in the concave of the porous composite material or adhering to the worn surface driven by the external force, and improving the flatness of the worn surface and the depth of the worn groove of the composite material. Therefore, the comprehensive properties of the graphene modified PTFE/PI porous materials have been improved.

\section{Conclusion}

(1) When graphene is embedded in the composite, its excellent thermal conductivity improves the thermal conductivity of the composite. Test results show that the thermal conductivity of graphene modified PTFE/PI composites is about 37\% higher than that of PTFE modified polyimide composites.

(2) The proper proportion of graphene components can effectively improve the mechanical properties of PTFE/PI composites. Tensile test shows that the tensile strength of composites is best when the proportion of graphene is about $0.5 \%$.

(3) With the increase of graphene ratio, the wear volume of graphene modified PTFE/PI shows a tendency to increase first and then decrease, the rigidity of graphene and the self lubrication of polytetrafluoroethylene will determine forms of the frictional properties with the change in the proportion of graphene and polytetrafluoroethylene. 


\section{Acknowledgements}

This work was financially supported by the National United Engineering Laboratory for Advanced Bearing Tribology (201708), Henan Natural Science Foundation Project (162300410086) and Henan province science and technology key project (172102210254).

\section{Reference:}

[1] WANG Zijun, LI Chunwei. Polymer Transfer Lubricating Film for Space Application[J]. BEARING, 2011(5):55-57.

[2] WANG Zijun. The Study of Oil-impregnated Micro Porous Polyimide Retainers Used for gyroscope Bearings[D]. HeFei:HeFei University of Technology, 2004.

[3] SUN Xiaobo, WANG Zijun. Study on Forming Process and Properties of Polypyromelliticimide retainer Material[J]. BEARING, 2016(11):37-41.

[4] C H Liu, X Y Chen, S N Jang, et al. High-speed wear lifetime analysis of instrument ball bearings[J]. Journal of Engineering Tribology,2009,223(3):497-510.

[5] Yan P X, Zhu P, Huang L J, et al. Study on tribological properties of porous polyimide containing lubricants[J]. Tribology, 2008, 28(3):272-276.

[6] A. Patnaik, A. Satapathy, N. Chand, et al. Solid particle erosion wear characteristics of fiber and particulate filled polymer composites: A review[J]. Wear, 2010, 268(1-2): 249-263.

[7] K. S. Novoselov, V. I. FafKo, L. Colombo, et al. A roadmap for graphene[J]. Nature, 2012, 490(7419): 192-200.

[8] JIA Yuan, YAN Hongxia, GONG Chao, et al. The Surface Modification of Graphene and Its Application in the Friction Field[J]. Materials Review, 2013, 27(5): 18-21.

[9] Y. J. Shin, R. Stromberg, R. Nay, et al. Frictional characteristics of exfoliated and epitaxial graphene[J]. Carbon, 2011, 49(12): 4070-4073.

[10] K. S. Kim, H. J. Lee, C. Lee, et al. Chemical vapor deposition-grown graphene: the thinnest solid lubricant[J]. ACS Nano, 2011, 5(6): 5107-5114.

[11] X. Z. Liu, Q. Y. Li, C. G. Lee, et al. Nanoscale friction and adhesion behavior for few-layer graphene[J]. Tribology\&Lubrication Technology, 2011, 67(11):12-14.

[12] D. Marchetto, C. Held, F. Hausen, et al. Friction and wear on single-layer epitaxial graphene in mufti-asperity contacts[J]. Tribology Letters, 2012, 48(1SI): 77-82. 